\title{
Parfum de chauvinisme ou résistance culturelle dans La Femme parfum d'Abdoulaye Élimane Kane
}

\author{
Dame KANE \\ Université Cheikh Anta DIOP - Sénégal \\ Reçu le 5 mars 2019 | Acceptéle 30 juin 2019
}

\begin{abstract}
RÉSUMÉ. Depuis les années trente, plusieurs œuvres de la littérature négro-africaine vont se se détacher progressivement de l'éloge de la métropole et matérialiser une forme de résistance assimilable au chauvinisme qui apparait, non seulement, tel un désir de reconnaissance et d'affirmation identitaire mais également, au-delà, comme un bouclier permettant de s'opposer ou d'atténuer l'influence des ondes culturelles venant d'ailleurs. La Femme parfum du romancier et philosophe sénégalais Abdoulaye Kane (2009), fait partie de ces textes qui, avec finesse et profondeur, expriment cet état de fait en s'intéressant aux qualités intrinsèques de communautés négro-africaines, souvent minoritaires, en exposant leurs valeurs puisées de la tradition dont elles continuent, non sans difficultés, à pérenniser les fondements et les contours dans un contexte particulier où la mondialisation tend vers une uniformisation culturelle au détriment de la diversité des modes de vie. Il s'agit, ici, en s'inspirant de l'approche sociocritique de Lucien Goldman dans Dieu caché (1956), d'analyser les indices d'un certain chauvinisme ou la manière dont la résistance culturelle s'organise dans ce roman. Ce qui nous permettra de cerner ses modalités, son mécanisme et sa portée.
\end{abstract}

Mots-clés : chauvinisme, conservatisme, culture, négro-africaine, parfum, résistance, Sinthiou

\begin{abstract}
Since the 1930s, several works of Negro-African literature have gradually become detached from the praise of the metropolis and materialize a form of resistance comparable to chauvinism that appears not only as a desire for recognition and affirmation of identity but also Beyond, as a shield to oppose or mitigate the influence of cultural waves from elsewhere. The Femme parfum of the Senegalese novelist and philosopher Abdoulaye Kane (2009), is one of those texts which, with finesse and depth, express this state of affairs by focusing on the intrinsic qualities of BlackAfrican communities, often minority, by exposing their These values are derived from the tradition of which they continue, not without difficulties, to perpetuate the foundations and outlines in a particular context where globalization tends towards a cultural standardization to the detriment of the diversity of lifestyles. It is here, inspired by the sociocritical approach of Lucien Goldman in Dieu caché (1956), to analyze the signs of a certain chauvinism or the way in which cultural resistance is organized in this novel. This will allow us to define its modalities, mechanism and scope.
\end{abstract}

Keywords: chauvinism,conservatism, culture, flavour, négro-african, résistance, Sinthiou 


\section{INTRODUCTION}

Sonnant ainsi comme un leitmotiv, la dimension culturelle du roman, ainsi le montre Kane (1983), est revendiquée, depuis quelques décennies par plusieurs romanciers qui critiquent ceux qui voudraient reléguer les cultures négro-africaines au second plan quoi qu'étant confrontées à des écueils multiples dont elles doivent faire face pour assurer leur pérennité. Ainsi la reconnaissance et l'affirmation de soi, de son identité culturelle dans un monde globalisé, sont-elles devenues des impératives de survie pour toutes les communautés menacées de disparition du fait surtout de l'hégémonie des grandes puissances. Une telle exigence a fini d'engendrer une forme de résistance culturelle que $l^{\prime}$ on pourrait assimiler au chauvinisme, repli exclusif sur soi. Ce prédicat qui sonne, de manière appuyée, le divorce avec toute vision afropessimiste, est, selon Dabla (1986), largement visible dans certains romans négro-africains francophones de ce début du XXIème siècle notamment dans La FemmeParfumde Kane (2009). Combinant une grande connaissance des cultures noires avec une écriture prolixe, d'une grande clarté et quasiment indemnes $d$ 'interférences linguistiques, ce romancier sénégalais nous offre une large vue d'un choc des cultures dont le champ est Sinthiou pathé.

Né le 6 octobre 1941 à Kayes au Mali et titulaire d'un doctorat d'Etat en philosophie, Abdoulaye Élimane Kane a été professeur au département de philosophie. La FemmeParfum, son cinquième roman, est publié en 2009 aux Presses Universitaire de Dakar. Dans ce roman il relate l'histoire de Kadia, une styliste de grande renommée surnommée la magicienne du fil, dont la dernière création («la femme-laser»), fait l'objet de beaucoup de convoitise du fait de sa fibre spéciale. Arrêtée et emprisonnée sans motif valable, Kadia s'installe dans un village nommé Sinthiou-Pathé ou village de Liberté. Soma le gardien du Bois Sacré lui fait part du secret de la fibre spéciale qui confère à ses créations toute leur authenticité et leur originalité. Local Africa Coton, une entreprise multinationale, voulant s'approprier du secret de cette fibre, use de toute sorte de subterfuge. La robe sera volée ensuite retrouvée et finalement vendue aux enchères. En revanche, "la Femme-Parfum», «la robe jumelle» de " la Femme-laser», est jalousement gardée par Kadia afin de reconquérir son identité de créatrice.

Plusieurs œuvres négro-africaines contemporaines se caractérisent par un contenu fort riche en substances culturelles. La Femme parfum n'échappe pas à ce constat. Ce qui justifie, dans une large part, son choix comme notre corpus d'analyse. C'est un roman qui s'inscrit dans une approche globale matérialisant le désir de l'auteur de forger une série d'œuvres liées les unes aux autres et formant un tout à la fois marqué par la diversité et l'unité des objets abordés dont la dimension culturelle en constitue le socle. Par ailleurs, il vient confirmer l'engagement de ce romancier négro-africain dans la défense et la revalorisation de nos biens immatériels souvent mal connus ou simplement méprisés. Il s'adresse ainsi « à tous les créateurs, individuels et collectifs qui luttent pour la liberté de création, la protection du patrimoine et une vie décente » (Kane, 2009).

\section{MÉTHODE}

La sociocritique, comme étude de la manière dont les mécanismes sociaux s'intègrent dans les œuvres, est une approche intéressante pour se pencher sur la culture et l'évolution des sociétés. À ce titre, nous nous focaliserons sur la vision de Goldman (1956) qui demeure l'une des plus grandes figures de cette discipline qu'il appréhende tel une analyse des données sociales, historiques, doctrinales mais surtout culturelles dans les œuvres littéraires. Il s'appuie sur le caractère hybride du roman pour mettre en exergue la dégradation des valeurs traditionnelles dans un monde dynamique qui tend irréversiblement vers l'uniformisation d'une certaine modernité «tueuse» de ce qui constituait le soubassement des sociétés anciennes dont les contemporains sont les héritiers.

Ainsi dans Dieu caché (Goldman, 1956), l'auteur étale les raisons qui font que le texte 
dépasse l'individu, le personnage comme entité isolée et plonge ses racines dans les profondeurs de la société dont l'écrivain en est un membre. Cette contribution va extraire, de notre corpus, des faits socioculturels qui seront analysés en rapport avec notre problématique. Ce qui pourrait aboutir à une certaine lumière sur les moyens de résistance culturelle face aux menaces tant endogènes qu'exogènes qui risquent de nuire aux traditions négro-africaines qui se métamorphose crescendo.

\section{RÉSULTATS ET DISCUSSION}

Du latin «conservatum» (Olivetti, 2003) signifiant conservé, le conservatisme désigne l'opinion ou l'état d'esprit de ceux qui refusent le progrès qu'il soit politique, culturel, social ou technologique. Aussi, Soma,personnage clé assumant pleinement son statut de villageois très conservateur, affiche, dans une large part, son penchant pour l'animisme face au Christianisme importé en Afrique par l'occident notamment au moment de la colonisation et à l'Islam qui s'est implantée grâce aux conquêtes arabes. Cette attitude spirituelle anti-progressiste apparait dans l'engagement de ce personnage à manifester en toute fierté son appartenance religieuse qui trouve son ancrage dans le sillage de la tradition :

C'est surement lui, avec toujours sur la tête un chapeau de paille. Il défend et pratique une religion traditionnelle dont le principe est l'accord avec la nature. C'est lui qui a créé ce domaine qu'il a appelé «le bois sacré» Il fait de la consultance à distance et à domicile, du bricolage et des croisements de plantes. (Kane, 2009, p.94)

La présence de ces pratiques s'explique, notamment, par le fait que l'Afrique a connu pendant des millénaires qu'une seule religion qui est l'animisme avec toutes les pratiques qui le caractérisent. C'est un héritage culturel qui apparait dans nos textes. Il est aussi à noter que les pratiques animistes, comme nous le fait comprendre Fakoly (2004), vont avec l'arrivée du christianisme, perdre progressivement leur importance et leur originalité.
Cette spiritualité occupe une grande place dans cet espace. En effet les personnages qui y sont impliqués jouent des rôles de premier plan dans La Femme parfum. Ils ne s'y intéressent pas forcément pour se protéger, accéder à des avantages ou pour nuire à certain, comme il est courant dans plusieurs sociétés. Mais elle permet surtout de concurrencer la médecine moderne et pérenniser des pratiques médicales traditionnelles qui reflètent aussi une certaines identité propre au habitant de Sinthiou Pathé.

À la question de savoir s'il faut livrer le secret de la fibre aux étrangers Pathé le fondateur du village, Billo de même que soma, Kadia et Gaspard répondent par la négative seul Charles est contre leur décision. Les habitants de Salémata et de Fongolembi observent ce conservatisme en refusant de vendre le masque bassari à Gaspard qui s'adresse à Charles :

Ecoute...lorsque nous sommes allés à Salémata tous les deux, nous avons d'abord feint de vouloir acquérir le fameux masque bassari qui est incontestablement une pièce remarquable. Il ne nous a pas été difficile de comprendre le refus des populations. $\mathrm{Tu}$ as vu également que si elles ont catégoriquement rejeté toute éventualité de le céder à un musée ou à un particulier même avec une contrepartie de sommes d'argent importantes. (Kane, 2009, p.208)

Ce masque est plus qu'un objet d'art, en réalité, il a une double fonction à la fois sociale et mystérieuse. Il contribue ainsi à l'harmonie du village. Ce que semble ignorer les étrangers notamment les Occidentaux qui travaillent pour les sociétés multinationales. C'est ce qui justifie, dans une large part, le refus sans réserve des villageois de vendre cet objet si symbolique et valeureux pour eux. À ce titre Charles rappelle aussi la réponse du chef de village "que nous restera $t$-il lorsque tout l'argent qui aura été acquis en le cédant sera épuisé ? »(Kane, 2009, p.208)

Dans une partie de La Femme parfum, les indigènes, comme Soma et Diarno, doivent résister à l'assaut de la culture étrangère, engager une véritable bataille contre cette nouvelle réalité qui mine leurs 
croyances traditionnelles.Mais pour saisir les menaces culturelles les plus profondes contre lesquelles les sociétés africaines doivent faire face pour maintenir leur spécificité, il nous faut examiner comment La Ferme parfum représente l'espace-ville.

L'espace urbain comme la ville de Dakar, se voit intégrer dans un jeu de parallélisme spatial qui l'oppose à la campagne en l'occurence Sinthiou Pathé qui, du fait de son conservatisme, de son hostilité vis-à-vis de tout ce qui est étranger à ses traditions, à son mode de vie, rejette toute modernité mal comprise et qu'incarnent les grandes villes. Cette interaction, entre les milieux urbain et rural, rendu possible par les activités des personnages qui y évoluent, se comprend mieux par une analyse séparée de ces deux entités d'une part la campagne qui, quoiqu'étant en posture de résistance culturelle, se métamorphose progressivement et d'autre part la ville apparaissant comme l'espace d'une modernité très problématique.

Le conservatisme des villageois, surtout les défenseurs de la tradition comme les personnes âgées, a permis d'en assurer la continuité. Ainsi est-il possible de voir les villageois continuaient d'observer les mêmes pratiques que leurs aïeux les ont léguées. Les règles protocolaires dans les cérémonies sont particulièrement respectées et aucun manquement n'est toléré. $M$. Chang se rend en fin de compte que "les habitants du village étaient aussi bizarres et fermés » (Kane, 2009, p.236) après avoir tenter à plusieurs reprises de découvrir le secret de la fibre du bois sacré :

Du matin au soir,...Ils sont très déçus, ils sont très mécontents. Pour eux, avec tous les cadeaux qu'ils ont envoyés, le travail qu'ils ont donné aux jeunes du village, en plus de tous les médicaments distribués et tout le reste qu'ils ne peuvent pas dire publiquement, les chefs de quartiers ne devaient pas refuser de leur dire comment est fabriquée la graine de coton... (Kane, 2009, p.236)

Le village n'est pas seulement caractéristique de son mode de désignation, nommé ou anonyme, mais aussi de son aspect physique et de sa culture. Pendant que la ville présente partout des constructions modernes, des bâtiments administratifs, des villas, des cités de fonctionnaires, le village, lui, conserve ses cases souvent à toit de chaume. Sur le plan proprement culturel, deux faits peuvent être observés: les éléments de la civilisation moderne y sont présents, ne serait-ce qu'au niveau de la religion chrétienne et de l'argent qui circule. Sinthiou Pathé présente de moins en moins les aspects d'une culture authentiquement pure, même s'il ne fait aucun doute que par rapport à la ville, il conserve encore des traits caractéristiques proches de l'Afrique précoloniale.

La femme parfum transpose bien la difficile conservation de la tradition, plus particulièrement, par les personnes âgées qui forment le cercle des sages qui gardent jalousement l'héritage des anciens. Chez Soma cet esprit conservateur qui rejette une partie de la modernité à l'occidentale, est largement dû à la menace que constituent les réalités qui prévalent dans l'espace urbain, contre la survie des traditions ayant pour lieu d'ancrage, l'espace rural. Cet attachement à soi se voit jusqu'au niveau de la production de biens divers, notamment, dans la fabrication d'articles comme les habits, tous les éléments qui $\mathrm{y}$ sont nécessaires proviennent du village et non de l'étranger :

Dans dix jours, cette dizaine de jeunes filles va se transformer en œuvres d'art vivantes et ambulantes sur les planches de ce lieu mythiques qu'est «La Toile arc-en-ciel ». Les dépliants touristiques ont porté ce nom aux quatre coins de la Terre. Ils vantent la régularité de ce rendez-vous, les mille couleurs de ses guirlandes, ses fumets de poisson et de merguez et ses exhalaisons d'arômes de jus de goyaves, de corossol, de papaye et cajou. (Kane, 2009, p.219)

À cela s'ajoute largement la créativité décrite comme la capacité des ces personnages à imaginer ou construire et mettre en œuvre des pratiques leur permettant de mieux défendre et faire connaitre la richesse de leur mode de vie pouvant inscrire sa marque dans la culture universelle. C'est l'art et la culture que les villageois essaient de valoriser et de promouvoir. Kadia possède une exploitation 
où des femmes travaillent la fibre et elle s'en sert pour ses créations. Il y a aussi l'existence d'ateliers de fabrication de galettes de mil au lait de fromage. Les femmes transforment les feuilles de "koondi » (tirées de "l'arbre de la mariée") mélangées avec des graines de cotonnier pour en faire du parfum. parfum contenu dans de petits flacons que Kadia a garnilarobe «Femme-parfum » (Kane, 2009, p.12).

Ce rejet, avec finesse, de la modernité peut nous permettre de mettre en relief l'opposition qui existe entre espace urbain et espace rural qui demeure dans une situation de résistance, face aux assauts de la culture urbaine fortement influencée par l'Occident. Dans cette tension entre ces deux entités, le milieu rural semble être la plus grande perdante. Sinthiou Pathé, se trouvant menacée culturellement, pourrait se désagréger de tous côtés. Le narrateur en est très conscient. Le pouvoir de l'argent n'est plus un sujet inconnu. Et la recherche du profil influence de plus en plus les comportements des ruraux :

La dernière nouveauté dans cette localité, décidément bien petite mais riche en événement, $c^{\prime}$ est l'offre faite par Local Africa Cotton aux villageois. Ils annoncent leur intention d'acheter la totalité de la récolte de coton, à un prix imbattable. La nouvelle a ébranlé certains jeunes... Ils ont même menacé leurs parents et les notables de réclamer leur part pour faire la transaction. (Kane, 2009, pp. 248-249)

Ainsi, si autrefois le pouvoir de l'argent n'avait pas la dimension qu'il a aujourd'hui au village, force est de reconnaitre que de plus en plus l'espace rural subit les effets de la recherche du profil de la majorité de ses habitants. Les jeunes sont la catégorie de personnages la plus touchée par ce goût effréné du profil et du matérialisme. Pour accéder à la richesse. Ils sont prêts à tout, même à sacrifier leurs communautés, leurs propres familles. Ce comportement de la nouvelle génération était ignoré des rustiques notamment des vieux qui se désolent d'une telle situation. Dans $L a$ femme parfum, les gardiens de la tradition critiquent l'attitude de certains jeunes égoïstes qui ne pensent qu'à se faire de l'argent au détriment de l'intérêt général. Soma et Diarno affiche leur conservatisme après la proposition de Local Africa Cotton d'acheter à un prix imbattable la totalité de la récolte $\mathrm{du}$ coton. Une proposition qui divise les jeunes du village et les anciens essaient de trouver un consensus :

La dernière nouveauté dans cette localité, décidément bien petite mais riche en évènements, $c^{\prime}$ est l'offre faite par Local Africa Cotton aux villageois. Ils annoncent leur intention d'acheter la totalité de la récolte de coton, à un prix imbattable... Le désaccord semble profond sur cette question... Il s'est mis à la tête des jeunes convaincus de la nécessité de résister aux offres de Local Africa Cotton. (Kane, 2009, p.249)

Les multinationales, qui représentent la culture de l'envahisseur, l'argent peut faire sauter tous les boucliers même les plus résistants. Cet état d'esprit, devenant de plus en plus général, contamine des communautés qui y étaient très réfractaires. Mais à Pathé, les jeunes, considérés comme les plus exposés aux apports de l'extérieur, tentent de tenir tête aux avances faites par cette grosse entreprise étrangère qui cherche à tout prix à découvrir le secret de la fibre magique afin d'en tirer beaucoup de profil en la commercialisant à grande échelle. Le conservatisme de cette communauté parait ,du coup, très solide grace à l'éducation des jeunes qui seront ainsi les futurs défendeurs $\mathrm{du}$ patrimoine culturel de ce village. Ce qui n'exclut nullement une certaine ouverture au monde extérieur et une tolérance notamment dans le domaine de la spiritualité.

Ensemble d'attitudes ritualisées et non profanes qui visent à mettre l'âme humaine en rapport avec la divinité, la religion est avant tout reconnaissance d'une entité supérieure à l'être humain, à qui ce dernier doit respect, obéissance et soumission, car de lui dépend sa destinée. Elle est comme le dit Amselle (2005) une pratique universelle. Le fait religieux étant établi comme un élément constant de la littérature négro-africaine, il convient maintenant d'en analyser ces enjeux quant à la matérialisation du chauvinisme spirituel qui se concrétise par la 
manière dont les pratiques spirituelles sont appréhendées dans ce roman de Kane (2009). Car si l'on peut affirmer une sorte de régularité dans l'évocation des pratiques religieuses (ou, magiques) à l'intérieur de $L a$ Femme parfum, on ne peut manquer de nous cristalliser sur la tolérance religieuse comme une forme d'identité culturelle approuvée, entretenue et conservée par une bonne partie des protagonistes de cette œuvre.

Sur ce plan les villageois, de convictions religieuses différentes, sont très ouverts et cohabitent ensemble dans la paix et l'harmonie. La preuve Billo qui était réticente au début quant à son mariage avec Charles un catholique finit par consentir à son union avec ce dernier. Tous les deux ont gardé leur religion. Gaspard un antillais qui pratique le culte Vaudou convole en noces avec Kadia qui est pourtant musulmane. Ils ont fait le mariage à la mode du Bois Sacré :

Soma a dit au début de la cérémonie, s'adressant aux deux couples: "puisque nous sommes tous des croyants, que chacun de vous se confie à Dieu, dans l'intimité de son cœur, le vœu de s'unir à l'autre dans les liens du mariage. Et prenez nous à témoins. (Kane, 2009, p.216)

Le syncrétisme spirituel apparait comme l'expression la plus élevée de cette tolérance religieuse que les habitants de Sinthiou Pathé comptent conserver à tout prix face aux menaces d'extrémisme qui viennent de l'étranger. On assiste à un mélange d'une multitude de sphères religieuses: christianisme, Islam et Animisme. Les croyants de ses religions célèbrent leurs fêtes ensemble et travaillent pacifiquement dans les mêmes structures où ils sont traités équitablement sans aucune discrimination. La société Mobfil s'est bien imprégné de cette réalité aussi organise-t-elle des soirées pour offrir des billets de pèlerinage à la Mecque et à Jérusalem :

L'entreprise de téléphonie mobile, Mobfil comme l'année précédente, organisait ce gala d'œuvres sociales en faveur de ses employés des différentes régions $\mathrm{du}$ pays. Cinquante billets d'avion DakarJeddah-Dakar pour le grand pèlerinage à la Mecque. Cinquante autres billets Dakar-Jérusalem-Rome-Dakar pour les catholiques de la Maison Mobfil. (Kane, 2009, p.55)

Le désir de raffermir les liens entre les membres d'une même communauté, explique ce procédé qui est plus qu'une politique d'entreprise mais contribue à étendre et à pérenniser la tolérance religieuse qui est vue comme une valeur essentielle faisant partie des files constitutifs du tissu culturel de cet espace ou la solidarité est d'une omniprésence indiscutable. La devise des villageois: "le village d'abord» (Kane, 2009, p.246), peut d'emblée laisser penser à un égocentrisme mais reflète surtout le lien fort, une solide solidarité, entre les membres de cet espace. Chacun considérant ses intérêts propres comme étant d'abord et avant tout ceux de la communauté qui est au dessus d'eux.

Sentiment de responsabilité et de dépendance réciproque au sein d'un groupe de personnes qui sont moralement obligées de s'entre-aider ,la solidarité est visible partout dans la communauté de Pathé. C'est un village où tout se fait par un consensus général. Le chef de Sinthiou se rappelle les raisons qui l'ont poussé à fonder ce village :

En faire un monde ouvert où l'on apprend à se respecter et à cultiver la liberté, la responsabilité et la solidarité. Il ajoutait souvent que cette idée est aussi vieille que le monde et aussi difficile à réaliser qu'elle est agréable à expliquer. Mais, qu'avec ce village de Liberté, quelque chose s'était mis en marche qu'il ne fallait pas arrêter. (Kane, 2009, p.190)

Dans La Femme parfum, la communauté y occupe un rôle important notamment quand il s'agit de défendre les intérêts de la collectivité. Si en Occident ainsi que dans les grandes villes africaines assez modernes, le groupe a cédé la place à l'individualisme, dans les villages traditionnels conservateurs comme Sinthiou dans cette œuvre, la communauté, bouclier permettant l'existence de toute résistance notamment culturelle, est au début et à la fin de cet espace rural dont les habitants considèrent que si l'individuest isolé il devient fragile. Ainsi pourrait-il perdre son identité aux dépens d'une nouvelle vision. Les femmes, à travers certaines de leurs 
activités, contribuent largement au renforcement de ce chauvinisme :

Parmi celles-ci, encore et toujours, un usage particulier des graines du même type de cotonnier. Dans ce quartier, les femmes fournissent à l'ensemble des habitants les quantités nécessaires à la satisfaction de leurs besoins en savons et pommades. Ils entendent se servir... se suffire avant de penser à l'extérieur. (Kane, 2009, p.244)

Ceci est largement la manifestation d'une grande et sincère solidarité cristallisée dans le village par et pour ses résidents c'està-dire à leur seul bénéfice. Elle tire ses racines $d^{\prime}$ une tradition qui considère que tous les membres d'une communauté sont des parents les uns liés aux autres par le sang ou par le jeu des alliances sociales. Résistants à l'individualisme caractérisant la modernité, Ses habitants défendent ces valeurs culturelles de manière à la fois tout aussi naturelle que désintéressée mais en dehors de toutes naïveté car étant totalement conscients des dangers qui guettent leur culture, leur mode de vie et qui pourraient nuire à leur identité et par conséquent à leur devenir.

\section{CONCLUSION}

La Femme parfum expose un univers cosmopolite dont les rapports des personnages entre eux et les réalités de leurs milieux sont doubles: dans un sens une résistance culturelle qui, face à une modernité à l'occidentale, se matérialise par la persistance des pratiques culturelles à Sinthiou Pathé et d'un autre coté par un chauvinisme qui se constate par une forme de repli sur soi et de défense des intérêts intracommunautaires. Il est aussi à noter une certaine corrélation entre des pratiques culturelles locales et étrangères qui ne sont pas présentes comme des diapositifs superposés, indépendants les uns des autres mais entretiennent, au contraire, des rapports de complémentarité créant du coup une ouverture et une diversité culturelles.

Par ailleurs, il apparait, de manière feutrée, que la vision des personnages varie selon que l'on soit originaire du nord ou habitant du village de Sinthiou pathé. Cette différence peut s'expliquer de plusieurs manières. Elle est d'ordre culturel, économique,politique ou social. Elle se reflète au niveau des comportements et des modes de vie. Cependant, résister par l'unique canal $\mathrm{du}$ conservatisme pourrait ne pas suffire comme le laisseraient penser plusieurs écrivains. Les communautés doivent trouver dans ce qu'ils ont déjà de quoi nourrir leur créativité pour réinventer de nouvelles formes de résistance culturelle qui raffermiraient l'ancrage, l'enracinement dans leur propre culture sans oublier de s'ouvrir à ce que le poète Senghor appelle les « apports féconds » de l'extérieur.

\section{REMERCIEMENTS}

Créé en août 1936, l'institut fondamentale d'Afrique noire, connu sous l'acronyme IFAN, continue de mener ses activités de recherche sur les cultures et civilisations négro-africaines sous l'impulsion de plusieurs laboratoires dirigés par des universitaires de chevron. Le Professeur Amade FAYE, spécialiste de la littérture africaine orale, fait partie des Enseignants chercheurs les plus illustres de cet établissement. Je le remercie pour son engagement dans le domaine de la recherche, ses enseignements, sa générosité dans le partage de documents et sa disponibilité.

\section{RÉFÉRENCES}

Amselle J.L. (2005), Branchements. Anthropologie de l'universalité des cultures, Paris, Champs FlAmmarion,.

Dabla J.J.S. (1986), Nouvelles écritures africaines. Les romanciers de la seconde génération, L'Harmattan.

Fakoly, D. (2004) L'origine négro-africaine des religions dites révélées, Paris, Éditions. Menaibuc.

Goldman L.(1955), Dieu caché, Paris, Gallimard.

Kane A.E.(2009) La femme parfum, Dakar,Presses universitaires de Dakar, pages12,94,208,250,236,219,248,249,216, $55,190,244$. 
Kane, M. (1983), Roman africain et tradition, Dakar, Les nouvelles Editions africaines.

Olivetti, E. et Francesco, (2003) Grand dictionnaire latin. 